\title{
A Simplified Motion Isolated Load Control for Multi-Channel Syncro- Loading System
}

\author{
Zhang Yanyan, Yang Zhidong \\ Harbin Institute of Technology, Institute of Electro-hydraulic Servo Simulation Test System, 92 West Dazhi Street, Harbin, China
}

\begin{abstract}
For the multi-channel syncro-loading (MCSL) test, the specimen may be driven by a particular motion of another position channel, representing an in-service duty cycle. The loading accuracy is largely affected by the specimen motion. To reduce the cross coupling from the motion, the cross coupling characteristics between multi-channels is analyzed, and a simplified motion isolation load control (MILC) algorithm is provided, in which the velocity and acceleration of the specimen, measured by the accelerometer, are added into the forward path of the loading control channel in order to drive the actuator to match the motion of the specimen. The test results indicate that the MILC algorithm can significantly improve the loading accuracy compared with the conventional loading control method.
\end{abstract}

\section{Introduction}

The multi-channel syncro-loading system driven by electro-hydraulic actuators is an important facility for the structure test, and it has been extensively applied in the aerospace, train, vehicle, and material fields [1-4]. During a test, it is necessary to simulate the multi-directional load on the specimen, and the loading accuracy is very important to evaluate the strength, lifetime and performance of the specimen. However the conventional control method, which controls each actuator independently[1, 3-5], can not usually reach the desired loading accuracy, because there is the strong cross coupling among the actuators. Especially, when controlling the position and force simultaneously, the force of one channel can be affected by the position of another channel seriously.

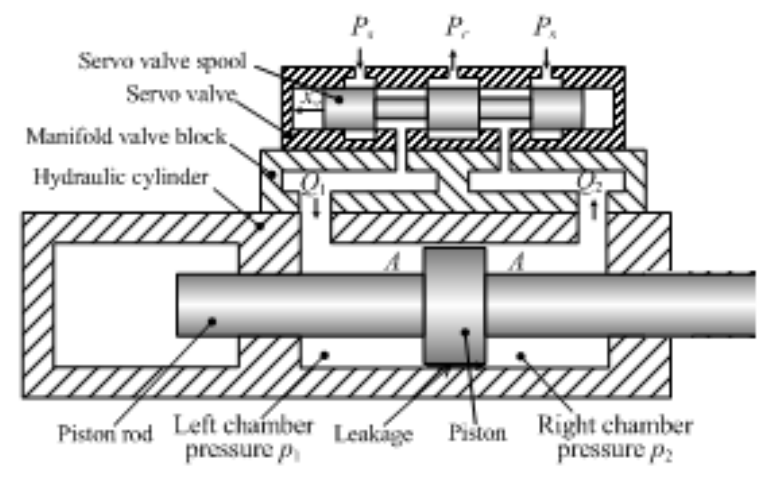

Actuator controlled by position
In the paper, to eliminate this loading coupling from the position channel, and improve the loading accuracy, the cross coupling characteristics between the position channel and the force channel is analyzed, and a motion isolated load control algorithm is proposed, which is then validated in the fatigue test of the train's bogie.

\section{The effect of motion on loading accuracy}

The multi-channel syncro-loading system usually includes more than one actuator, which may be controlled by position or force. There are some cross coupling between the force controlled actuator and the position controlled actuator.

Figure 1. Actuators controlled by positon and force in MCSLs. 
The actuator is mainly composed of the highfrequency electro-hydraulic servo valve and the double rod asymmetric hydraulic cylinder, the model of which is shown as Fig. 1. For the force controlled actuator, the mathematical mode can be derived.

The load flow supplied by the valve is the function of the spool displacement and the load pressure drop:

$$
Q_{L F}=K_{q F} \cdot X_{v F}-K_{c F} \cdot P_{L F}
$$

where, $Q_{L F}$ is the load flow, $P_{L F}$ is the load pressure drop, $K_{q F}$ is the linearized flow coefficient, $K_{c F}$ is the linearized flow-pressure coefficient and $X_{\mathrm{vF}}$ is the spool displacement of the servo valve.

Taking leakage and compressibility into consideration, the load flow of the hydraulic cylinder is:

$$
Q_{L F}=A_{F} s Y_{F}+\left(C_{t c F}+\frac{V_{t F}}{4 \beta e} s\right) P_{L F}
$$

where, $A_{F}$ is the piston area, $Y_{F}$ is the piston displacement, $C_{t c F}$ is the total leakage coefficient of the cylinder, which is comprised of the internal leakage and external leakage, $\mathrm{V}_{t F}$ is the total volume of the oil in two cylinder chambers, $\beta e$ is the bulk modulus of the fluid.

The output force of hydraulic cylinder is:

$$
\begin{aligned}
A_{F} P_{L F} & =m_{F} s^{2} Y_{F}+B_{c F} s Y_{F}+F \\
F & =K_{L}\left(Y_{F}-Y_{P}\right)
\end{aligned}
$$

where, $m_{F}$ is the equivalent weight of the piston, $B_{c F}$ is the viscous damping coefficient of the piston, and $F$ is output force of the hydraulic cylinder. The piston displacement $Y_{p}$ of the actuator controlled by position can affect the output force, as shown in Eq.4, and it can be seen as the disturbance variable.

Combining Eq.1- Eq.4,we can get the Transfer Function (TF) from $Y_{p}$ or $X_{v F}$ to $F$, as illustrated in Fig .2.

It can be further simplified as Fig. 3.

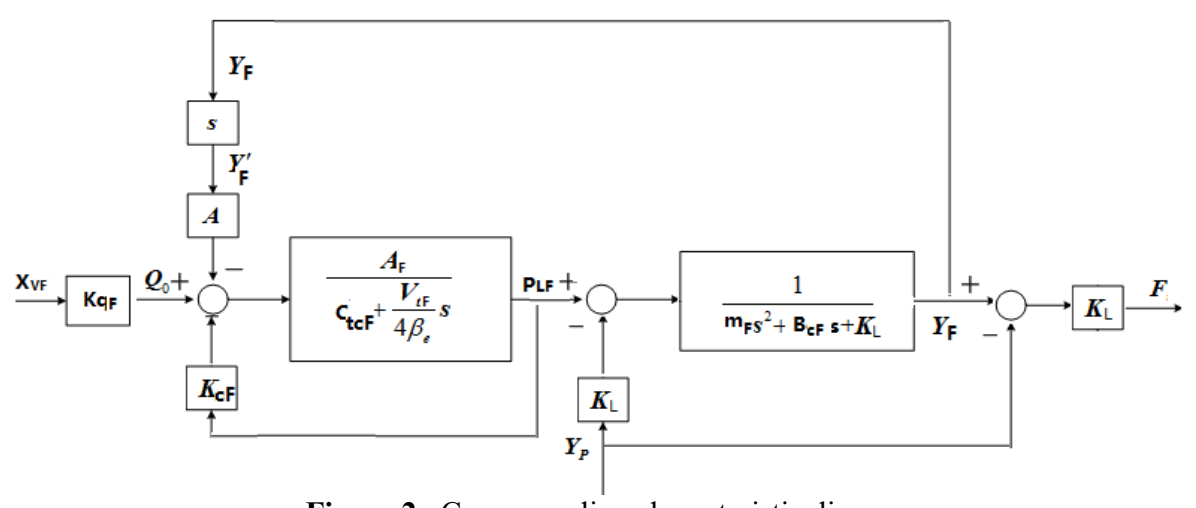

Figure 2. Cross coupling characteristic diagram

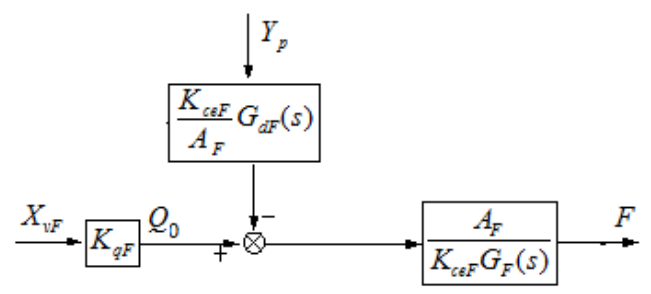

Figure 3. Simplified cross coupling characteristic diagram

Supposing $X_{v F}=0$, the output $F$ derived from $Y_{p}$ is the coupled force $F_{d}$, which can be seen as the loading error, and undesired. It can be shown as:

$$
F_{d}=Y_{p} \cdot G_{d F} / G_{F}
$$

where

$$
\begin{aligned}
G_{d F}(s)= & \frac{A_{F}^{2}+K_{c e F} B_{c F}}{K_{c e F}}\left[\frac{m_{F} V_{t F}}{4 \beta_{e}\left(A_{F}^{2}+K_{c e F} B_{c F}\right)} s^{2}\right. \\
& \left.+\left(\frac{B_{c F} V_{t F}}{4 \beta_{e}\left(A_{F}^{2}+K_{c e F} B_{c F}\right)}+\frac{m_{F} K_{c e F}}{A_{F}^{2}+K_{c e F} B_{c F}}\right) s+1\right] S
\end{aligned}
$$

$$
\begin{aligned}
G_{F}(s)= & \frac{m_{F} V_{t F}}{4 \beta_{e} K_{F} K_{c e F}} s^{3}+\left(\frac{B_{c F} V_{t F}}{4 \beta_{e} K_{F} K_{c e F}}+\frac{m_{F}}{K_{F}}\right) s^{2} \\
& +\left(\frac{V_{t F}}{4 \beta_{e} K_{c e F}}+\frac{B_{c F} K_{c e F}+A_{F}^{2}}{K_{F} K_{c e F}}\right) s+1
\end{aligned}
$$

According to Eq.5, it can be seen that the coupled force $F_{d}$ is determined by the velocity, acceleration and jerk of the position system. And the jerk is just a temporary result, the key elements are only velocity and acceleration.

\section{Simplified MILC algorithm}


For the velocity and acceleration of the specimen are the key elements for the coupled force $F_{d}$, in order to reduce the influence of the coupled force on the dynamic loading precision of the loading system, the simplified MILC (Motion Isolated Load Control) is proposed, in which the velocity and acceleration of the position controlled channel is feed forward on the force controlled channel to compensate influence of the coupled force, as shown in Fig. 4. If the high frequency noise is introduced by the acceleration feed forward, a low pass filter before the high pass filter. The compensation rate is limited by the maximum velocity of the actuator.

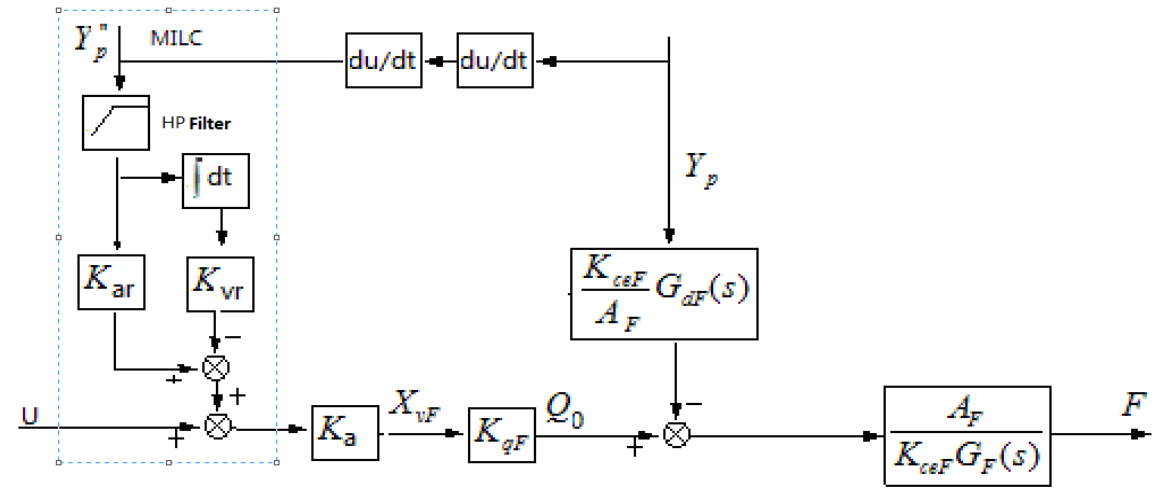

Figure 4. MILC using Acceleration and Velocity

In the simplified MILC, two hardware additions are required compared to the conventional loading control method:

(1) An accelerometer, measuring the acceleration along the axis of movement of the MILC-controlled actuator, must be attached on the specimen.

(2) A compliant element, for example a rubber bush, should be fitted between the MILC-controlled actuator and the specimen. This provides some mechanical isolation between the specimen and the actuator.

The motion of the specimen, in terms of the velocity and acceleration, is obtained though the accelerometer. Then the velocity and acceleration signals are added into the forward path of the loading channel in order to drive the actuator match the motion of the specimen.

Besides any remaining mismatch between the actuator and specimen motion causes a loading error. The size of this error is directly related to the stiffness of the compliant element. The lower stiffness is better, but reducing the stiffness will have an effect on the performance envelope of the loading system. For example, the actuator is more likely to run out of the stroke for high load due to the extra movement of the compliant member.

\subsection{Tuning velocity feed forward}

Give a small or zero loading command to the actuator and excite the specimen with a high amplitude, low frequency motion (e.g. $2 \mathrm{~Hz}$ ), an unwanted loading variation will be seen. This is mainly related to the velocity of the specimen, which is the dominated factor at the low frequency.

Increase the velocity gain until the amplitude of the loading variation reaches the minimum. Theoretically, the optimal value for the velocity gain is the reciprocal of the maximum velocity of the actuator.

The 2nd order high pass filter in the method normally needs no adjustment. It affects the estimation of the velocity from the acceleration measurement as follows:
The cut-off frequency should be well below (e.g. by a factor of 10) the minimum frequency of interest to minimize phase distortion for the generated velocity. And a lower damping will cause that the phase distortion diminishes more rapidly as the frequency increasing, especially for the frequency greater than the cut-off frequency, but there is more signal amplification at the cut-off frequency. And you may find there is a small loading variation at the frequency similar to the cut-off frequency of the high pass filter. This can be removed by increasing the PID integral gain, or by increasing the cutoff frequency and/or damping.

\subsection{Tuning acceleration feed forward}

Keep a small or zero loading command for the actuator and excite the specimen with a high frequency motion $(10 \mathrm{~Hz}$ or greater), an unwanted loading variation will be seen; if the velocity gain has been adjusted correctly, this variation will mainly be related to the acceleration of the specimen.

Start the acceleration gain with 0.001 times the velocity gain. Increase the acceleration gain until the loading variation reaches the minimum.

\section{Experimental studies}

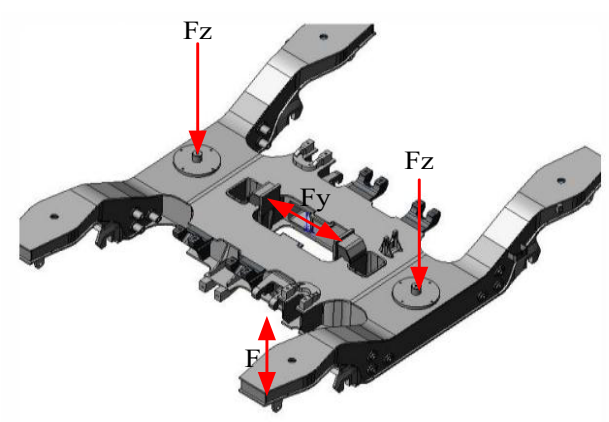

Figure 5. The layout of complex loading to bogie 
As the proof of the MILC, the fatigue test of the train's bogie is carried out according to the criteria in 《UIC6154-2003_20OR_20》 ${ }^{[6]}$. The loading channels controlled by the force, which is definited in Fig.5, include two vertical forces $F z$ and one longitudinal force $F y$. Meantime, there is another position channel to simulate the road irregularity.

Some experimental results are demonstrated in Fig.6, the feedback values of two vertical force loading channels and one position channel are recorded. During the test, the velocity and acceleration feed forward doesn't work at first, which is equivalent to the conventional loading control method, then the velocity and acceleration feed forward gain is adjusted, and the MCSL method works. It can be seen that, the jitter of the loading force has occurred at the peak of the half sinusoidal wave of the position channel for the conventional method. Adjusting velocity and acceleration feed forward gains, the jitter disappears gradually until the satisfied loading accuracy is obtained.
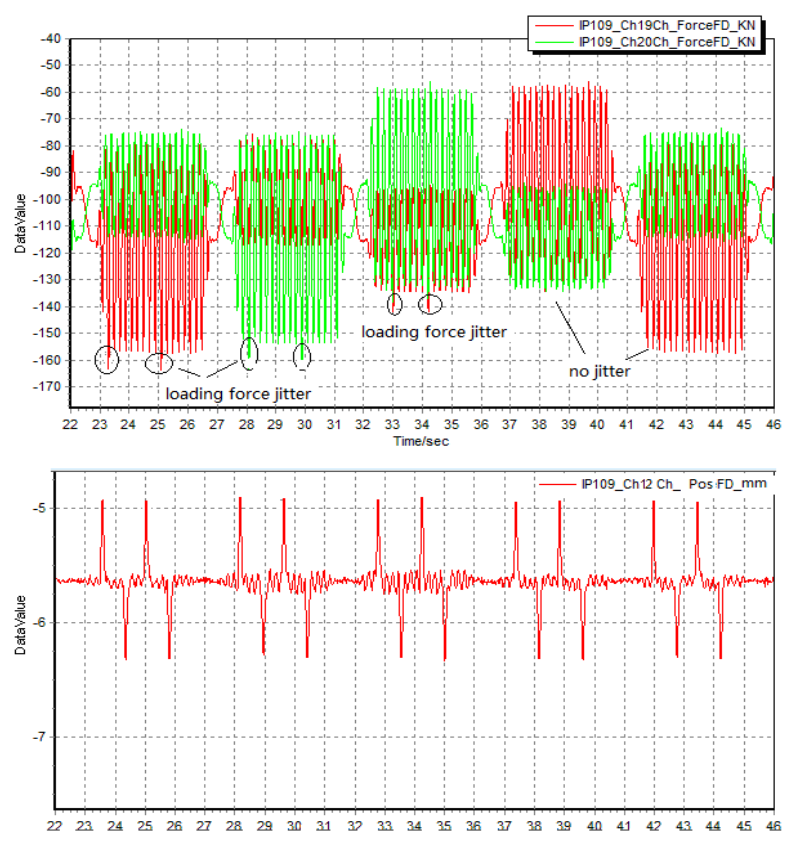

Figure 6. Results for the fatigue test
To eliminate the loading coupling from the motion of the position channel, and improve the loading accuracy, the cross coupling characteristics between multi-channels in the syncro-loading test is analyzed, and a simplified motion isolated load control algorithm is provided, in which the velocity and acceleration of the specimen are added into the forward path of the loading control channel in order to drive the actuator to match the motion of the specimen. Results of the train's bogie fatigue test show that the algorithm can significantly improve the loading accuracy compared with the conventional loading control method.

\section{Acknowledgment}

The author wishes to acknowledge contribution to some tests in this paper from Dr. Liu Weiya of the National Engineering Lab for High Speed Railway in Qingdao.

\section{References}

1. Dong Bo. Research on Application of Multi-channel Syncro-loading System in Strength Test of Power Bogie Frame. Railway Quality Control, 10, 13-15 (2007).

2. Yan Junmao. Vehicle Engineering(Third Edition). Beijing. China Railway Publishing House. 213-254 (2007)

3. Li Jupeng, Wei Wenjun. Research on Electrohydraulic Servo Loading System. Gansu Science and Technology, 21( 3) , 97-98 (2005)

4. Sui Jiefei. Design and Implementation of Electrohydraulic Servo Loading System of Distributed Rudder. Civil Aircraft Design and Research, 38( 2), 26-31 (2011)

5. Lu Lin,Wang Jun Zheng,Chen Cai Ke,Wang Li Tao. Application of Electro-hydraulic Force Control System in Structure Intensity Experimental Facility. Chinese Hydraulics \& Pneumatics, 8, 57-59 (2004)

6. UIC615-4-2003_20OR_20. Motive power unitsBogies and running gear-Bogie frame structure strength tests.UIC. (2003)

\section{Conclusions}

